\title{
Predictors of endoscopic prophylaxis for rebleeding in esophageal varices combined type 2 gastroesophageal varices
}

\section{Yuan Nie}

First Affiliated Hospital of Nanchang University https://orcid.org/0000-0001-7367-2359

\section{Mei-Chun Jiang}

Nanchang Number 9 Hospital

\section{Yue Zhang}

First Affiliated Hospital of Nanchang University

\section{Si-Zhe Wan}

First Affiliated Hospital of Nanchang University

\section{Cong Liu}

First Affiliated Hospital of Nanchang University

Xuan Zhu ( $\nabla$ waiyongtg@163.com )

First Affiliated Hospital of Nanchang University

\section{Research article}

Keywords: gastroesophageal varices, endoscopic treatment, secondary prophylaxis, rebleeding

Posted Date: December 4th, 2019

DOI: https://doi.org/10.21203/rs.2.18037/v1

License: () (1) This work is licensed under a Creative Commons Attribution 4.0 International License. Read Full License 


\section{Abstract}

Background and Aim Decompensated cirrhosis patients with esophageal varices (EVs) combined type 2 gastroesophageal varices (GOV2) have rebleeding risk after endoscopic prophylaxis. This study was performed to evaluate the preventive efficacy of endoscopic treatment in patients with EVs combined GOV2, and seek out the risk factor of rebleeding.

Methods We performed a single-center, observational, retrospective study of patients with EVs combined GOV2 extracted from hospitalization. The biochemical examination, demographics, endoscopic performance and treatment methods of patients undergoing endoscopic treatment were collected. All patients were observed until 1-year, or loss to follow-up. The predictors parameter of rebleeding were analyzed by logistic regression analysis

Results A total of 124 patients underwent endoscopic treatment, including $2(1.6 \%)$ patients failed to stop bleeding, 19 (15.3\%) patients loss to follow-up. 103 patients were observed until 1-year, including 11 (10.7\%) patients had rebleeding. The results had indicated EVs+GOV2 with GOV1, Age, PTA, Bilirubin, Platelet count, Child-Pugh, MELD were associated with rebleeding at 1-year in patients with EVs combined GOV2. Multivariate logistic analysis defined that Age, EVs+GOV2 with GOV1, Bilirubin, PTA were independent risk factor for rebleeding.

\section{Introduction}

Bleeding from gastroesophageal varices (GOVs) is a common and severe complication of liver cirrhosis and portal hypertension. It occurs in $~ 50 \%$ of patients with cirrhosis with an incidence of $10 \sim 15 \%$ each year $(1,2)$. Although the proportion of gastric varices (GVs) is lower than esophageal varices (EVs), the rebleeding and mortality of GVs is higher than $\mathrm{EVs}(3-5)$. The most common type of GV is GOV type 1 (GOV1), which accounts for about $70 \%$, followed by GOV type 2 (GOV2) at $21 \%$, isolated gastric varices 1 type (IGV1) at $7 \%$, and isolated gastric varices 2 type (IGV2) at $2 \%(6)$. However, the highest rebleeding type of GVs is IGV1, which has $78 \%$ of bleeding rate, followed by GOV2 bleeding rate of $55 \%$, and GOV1 and IGV2 of $10 \%(3)$.

Prognosis is an essential part of the assessment of any disease. Previous study has indicated risk of bleeding of gastric varices (1-year risk:10-16\%; 5-years risk:44\%) is connected with many factors, such as the subtype of GVs, Child Pugh score, hepatocellular carcinoma (HCC), red spots(3,6-8). These study about prognosis of rebleeding in GOVs patients is mostly concentrated on IGV1, however, the study in GOV2 is limited. At present, the endoscopic treatment is optimal important treatment of preventing rebleeding of GOVs, including endoscopic variceal sclerotherapy (EVS), endoscopic band ligation (EBL), endoscopic variceal obturation (EVO), and combined therapy(9). GOV2 is a type that more prone to bleeding, but study about the treatment effect and prognosis of rebleeding in GOV2 patients after endoscopic secondary prophylaxis treatment is uncertain. Therefore, we reigned this study with the 
purpose to seek out the risk factor with rebleeding in GOV2 patients after endoscopic secondary prophylaxis treatment in order to guide clinical practice.

\section{Patients And Methods Study design}

This was a single center retrospective observational cohort conducted at a large tertiary level public hospital in the South China between January 2009 and December 2018. Study protocol was approved by the institutional ethics committee of First Affiliated Hospital of Nanchang University. Informed written consent was obtained from all the study participants.

\section{Study population and setting}

The study cohort included all hospitalized patients aged $\geq 18$ years of EVs combined GOV2 by gastroscopic diagnosis with endoscopic secondary prophylaxis treatment. Exclusion criteria: No bleeding history; malignant tumors of extrahepatic origin; varices caused by non-cirrhotic causes such as the cavernous transformation of the portal vein; patients with TIPS and BRTO; Surgical periorbital vascular disconnection and other patients with shunt and devascularization; clinical data are not complete. All patients were treated following accepted recommendations and guidelines after admission to the hospital, and they were followed up until death or 1 year.

\section{Definitions.}

The presence of liver Cirrhosis was diagnosed according to clinical, biochemical, and radiological parameters; the presence of ascites, hepatic encephalopathy (HE), and/or signs of portal hypertension; ultrasonography; variceal bleeding; and even histology at hospital admission. Active bleeding: spurting at the variceal veins or active oozing; or varicose veins showing signs of recent bleeding (white/red thrombus or varicose veins covered with clots); or in the absence of other sources of bleeding, blood is visible in the stomach(10). Hemostasis failed: death occurred within 5 days of intervention, hematemesis $\geq 2$ hours after endoscopic treatment, hemoglobin decreased by $>3 \mathrm{~g} / \mathrm{dl}$ at 24 hours without blood transfusion, hemorrhagic shock(11). Rebleeding: hematemesis and/or melena reappear after 5 days of active bleeding(11). Renal impairment: creatinine levels $>1.5 \mathrm{mg} / \mathrm{dL}$ in patients with no kidney disease or $>50 \%$ in patients with previous kidney disease(12). The Child-Pugh score was calculated according to TBIL, albumin, international normalized ratio (INR), ascites status, and degree of HE. The MELD score was calculated using the formula: $3.78 \times \operatorname{Ln}(\mathrm{TBIL} \mu \mathrm{mol} / \mathrm{L})+11.2 \times \operatorname{Ln}(\mathrm{INR})+9.57 \times \operatorname{Ln}$ (creatinine $\mu \mathrm{mol} / \mathrm{L})+6.43 \times$ (constant for liver disease etiology $=0$, if cholestatic or alcoholic, otherwise $=1)(13)$. The count of MELD-Na was performed by using MELD+1.59 $\times[135-\mathrm{Na}(\mathrm{mmol} / \mathrm{l})](14)$. 


\section{Study protocol}

Patients with EVs combined GOV2 by gastroscopic diagnosis with endoscopic secondary prophylaxis treatment were enrolled in the study. During hospitalization, data were collected and compiled regarding demographic profile, history, clinical features, presence of other comorbidities, etiology of cirrhosis, type of decompensation and number of complications, endoscopic performance, and blood laboratory parameters at admission. Patients were followed for 1 year to look for survival situation. Patients with incomplete follow up at 1 year were not included in the final analysis of corresponding time.

\section{Statistical analysis.}

Statistical analyses were performed using SPSS software version 23.0 (SPSS Inc., Chicago, IL). Continuous variables were tested by the Kolmogorov-Smirnova method for normality test. The continuous data with the normal distribution was described by the mean \pm standard deviation, and compared by using Student's t-test. The continuous data without the normal distribution was described by the median (interquartile range [IQR]), and compared by using Mann-Whitney U-test. Categorical variables were described as frequency (percentage [\%]), and were compared by using Chi-squared analysis or Fisher's exact test. Logistic regression analysis was employed to demonstrate the predictors for the rebleeding rate of patients with EVs combined GOV2. All variables that were found to be associated with rebleeding $(P<0.10)$ were included as candidate variables in a forward conditional stepwise logistic regression analysis to identify independent predictors for the rebleeding of patients with EVs combined GOV2. All statistical tests were two-sided, and a value of $p<0.05$ was considered statistically significant.

\section{Results}

\section{Baseline characteristics}

As can be seen from Figure 1, 228 hospitalized patients with EVs combined GOV2 from January 2009 to December 2018 in this retrospective study. 22 patients were excluded:6 patients underwent TIPS, 12 patients underwent surgical shunt, 4 patients were cavernous transformation of portal vein, 2 patients were Budd-Chiari syndrome, and 1 patient was combined with gastric cancer. Of the 124 patients, 76 patients were $E V+G O V 2$, and 48 patients were $E V+G O V 1+G O V 2$. Considering GOV2 often combining with GOV1, therefore, the clinical features of 124 patients was also divided into EV+GOV2 without GOV1 groups, EV+GOV2 with GOV1. Of the 124 patients, 76 patients were EVs+GOV2 without GOV1, and 48 patients were EVs+GOV2 with GOV1. A total of 124 patients, median (interquartile range): 49 (42.2557.50). Majority of the patients were male (95/124, 76.6\%). Demographic, and biochemical characteristics of the study population are outlined in Table 1 . The main causes were hepatitis virusassociated cirrhosis (64.5\%). There were no significant differences in age, gender, creatinine, albumin, PTA, INR, bilirubin, hemoglobin, platelet counts, MELD score, MELD-Na score, Child-Pugh classification, 
HCC, ascites, HE, splenectomy between the EVs+GOV2 with GOV1 groups and EVs+GOV2 without GOV1 (all $P$ value $>0.05$ ).

\section{Endoscopic features of endoscopic prophylaxis in patients with EVs combined GOV2}

Majority of the patients with EVs combined GOV2 had red spot (111/124, 89.5\%), communicating branch (107/124, 86.3\%). EBL was mainly performed in patients with EVs combined GOV2 (95/124, 76.6\%), followed by EVS+EVO (11/123 8.9\%), EBL+EVS+EVO (6/124 4.8\%), EBL+EVO, EBL+EVS (3/124 2.4\%), EVO (1/124 0.8\%), EVS (6/124 4.8\%). There was no statistical difference between the EVs+GOV2 with GOV1 groups and EVs+GOV2 without GOV1 of endoscopic erythema, traffic branch, active bleeding, thrombus, variceal diameter, and distance from the incisors (all $P$ value $>0.05$ ).

\section{Comparison between the rebleeding group and the non-rebleeding group in patients with EVs combined GOV2}

124 patients underwent endoscopic treatment for secondary prophylaxis, including 2 patients hemostasis failure and 19 patients were loss to follow-up. Therefore, a total of 103 patients was enrolled in final research. According to whether there is bleeding within 1-year, 103 patients were divided into no bleeding group ( $n=92)$ and bleeding group $(n=11)$. The analysis showed that $E V+G O V 2$ combined GOV1 $(P=0.043)$ and age $(P=0.039)$, PTA $(P=0.019)$, bilirubin $(P=0.033)$, Child-Pugh grade $(P=0.009)$, MELD score $(P=0.044)$ were associated with rebleeding in patients with $E V s$ combined GOV2. By Multivariate logistic regression analysis, age (OR:1.186, 95\% Cl: 1.050-1.340, $\mathrm{P}=0.006)$, the variceal classification of EV+GOV2 with GOV1(OR: $3.063,95 \% \mathrm{Cl}$ : 1.560-6.184, P =0.024), bilirubin (OR:1.088, 95\% Cl: $1.022-$ 1.158, $\mathrm{P}=0.009)$, and PTA ((OR:1.109, 95\% Cl: 1.043-1.288, $\mathrm{P}=0.038)$ were an independent risk factor for rebleeding within 1-year. Among them, variceal classification was the most significant effect of EV+GOV1+GOV2.were the independent predictive factors for rebleeding within 1-year.

\section{Discussion}

Variceal bleeding is still one of the common serious complications in patients with liver cirrhosis. Even though with the development of medicine and endoscopic therapy, GVs accounts for $14-36 \%$ of acute variceal bleeding, and have a high rate of rebleeding (38\%-89\%) and mortality rate $(11 \%-20 \%)(15-17)$. According to Sarin classification, although the frequency of GOV2 is lower than GOV1, the rebleeding risk of GOV2 is significantly higher than GOV1. Prognosis is an essential part of the assessment of rebleeding. It is not only the basis for the information that a physician provides to the patient, but is also the basis for any decision-making process. As best known to us, EVs combined GOV1 has a high detection rate and is widely studied, but EVs combined GOV2 has limited research $(18,19)$. This study intends to analyze the endoscopic treatment effect and risk factors of rebleeding at 1-year in order to identify high-risk patients with rebleeding and take corresponding treatment measures to improve the prognosis. Considering EVs+ GOV2 is often accompanied by GOV1 in clinical, therefore, the clinical features and endoscopic features was divided into two groups (EVs+GOV2 without GOV1, EVs+GOV2 
with GOV1, respectively) and was compared, because no studies directly compare the clinical and endoscopic features between EVs+GOV2 without GOV1 and EVs+GOV2 with GOV1).

In our study, the causes of both groups were mainly hepatitis virus related cirrhosis, which is consistent with the epidemiological characteristics of cirrhosis in China. 124 patients underwent secondary prophylaxis, among which $2(1.6 \%)$ patient suffered hemostasis failure, $19(15.3 \%)$ patients were lost to follow-up, 11 (10.7\%) patients suffered rebleeding within 1-year. As is expected, the rebleeding incidence of patients underwent endoscopic prophylaxis was similar with other study $(16,17)$. In Dong XJ's study, there are 29 patients with EVs+GOV2, that 3 patients (10.3\%) were hemostatic failure, and 7 patients (24.1\%) were rebleeding within 6 weeks underwent EBL. The incidence of rebleeding of the patients in Dong XJ's study is higher than our study, maybe be caused by the higher rates of liver disease severity patients in Dong XJ's study(20). No statistical differences were found in endoscopic characteristics and clinical features between EVs+GOV2 without GOV1 group and EVs+GOV2 with GOV1 groups. Multivariate logistic regression analysis identified age, EVs+GOV2 with GOV1, bilirubin, PTA were a risk factor for 1years rebleeding in patients with EVs +GOV2. An increasing number of studies have been focused in predict esophageal and gastric variceal bleeding. In Xu L's study showed that PTA>18s (OR $=62.83$, 95\% Cl:9.39-420.56) and moderate/severe ascites (OR $=11.35,95 \% \mathrm{Cl}: 1.93-66.7)$ were independent risk factors for early rebleeding after endoscopic treatment(21). Kim SJ's study had indicated the Child-Pugh $\mathrm{C}$ was an independent risk factor for rebleeding in GVs patients(22). Our study showed that the ChildPugh score was associated with rebleeding in EVs+GOV2, but the Child-Pugh score was revealed that have not associated with rebleeding by multivariate logistic regression. The inconsistent conclusion may be related to the small number of Child-Pugh $C$ patients, the influence of albumin infusion on ascites degree and albumin level in some patients during treatment in our study. The blood provision of GOV1 and GOV2 is different, suggesting elevated portal vein pressure when present at the same time. Our study had found that patients with EVs+ GOV2 with GOV1 had an increased risk of rebleeding at 1-years and were independent risk factors $(\mathrm{OR}=31.063,95 \% \mathrm{Cl}$ : $1.560-618.4)$. Kang $\mathrm{EJ}$ et reported the rebleeding incident of patients with liver cancer $(P<0.001)$ and GOV2 $(P=0.009)$ was significantly increased at 1year when comparing the prognosis of 127 GVs patients (GOV1:GOV2: IGV $=56: 61: 10$ ) who had been treated with tissue glue for preventing rebleeding(23). A retrospective analysis by Jun $\mathrm{CH}$ et al reported $455 \mathrm{GVs}$ patients after endoscopic treatment showed that there was no different in GOV1, GOV2 groups rebleeding for 1-year(24), however, the comparing between EV+GOV2 without GOV1 group and EV+GOV2 with GOV1 was not completed. We found there was significant difference in rebleeding incident at 1-year between EV+GOV2 without GOV1 group and EV+GOV2 with GOV1 group. Our results indicated that combined with GOV1 may had influence in GOV2 of rebleeding. Mishra et al had reported that the MELD score $>18$, active bleeding at endoscopy are the risk factors of 6-weeks rebleeding and morbidity in GOV2 patients(25). Chen WT et had recently reported that MELD score was associated with rebleeding in GVs at 1-month (OR $=1.2,95 \% \mathrm{Cl}: 1.1-1.4)$ after endoscopic treatment(26). We showed that the MELD score was associated with rebleeding in patients with EVs+ GOV2, but it was not an independent risk factor and may be related to the low MELD score of patients in this study. 
Platelet counts could serve a noninvasive index to predict the severity of varices, and the decreasing of platelets can indirectly reflect increased portal pressure $(27,28)$. In this study, the platelet level in the rebleeding group was significantly lower than non-rebleeding group, however, no significant correlation was found in multivariate analyses. There were 1 patient in the rebleeding group had underwent splenectomy, that may have an effect on this result.

This study also has several limitations. First, as a single-center retrospective cohort research, the study may have a hereditary limitation and Some patients were loss to follow-up, which may result in the selection bias. Second, hepatic venous pressure gradient (HVPG) was not measured in all patients in the study due to limitations such as patient economy and acceptance of invasive examinations. Lastly, our study mainly focused on EBL treatment, but the different endoscopic treatment methods was no considered.

In conclusion, Endoscopic treatment is effective treatment of EVs combined GOV2. Age, EV+GOV2 with GOV1, bilirubin, PTA were independent risk factor for rebleeding in EVs combined GOV2 after endoscopic prophylaxis. Combining with GOV1 may had negative effect in patients with EVs +GOV2.

\section{Declarations}

\section{Author Contributions}

MCJ, YN and YZ contributed equally to this study. MCJ, and YN designed and wrote the manuscript, YZ collected the data, SZW and CL analysed the data, XZ critically revised the manuscript.

Funding: This study was supported by the National Natural Science Foundation of China (grant number: 81960120) and the "Gan-Po Talent 555 Project of Jiangxi Province.

Conflict of Interest statement: The authors declare that there are no conflicts of interest.

\section{Acknowledgements}

We would like to thanks the National Natural Science Foundation of China and the "Gan-Po Talent 555" Project of Jiangxi Province for the economic support.

\section{References}

1.Garcia-Tsao G, Sanyal AJ, Grace ND, Carey W, Practice Guidelines Committee of the American Association for the Study of Liver D, Practice Parameters Committee of the American College of G. 
Prevention and management of gastroesophageal varices and variceal hemorrhage in cirrhosis. Hepatology. 2007;46(3):922-38.

2.North Italian Endoscopic Club for the S, Treatment of Esophageal V. Prediction of the first variceal hemorrhage in patients with cirrhosis of the liver and esophageal varices. A prospective multicenter study. The New England journal of medicine. 1988;319(15):983-9.

3.de Franchis R, Primignani M. Natural history of portal hypertension in patients with cirrhosis. Clinics in liver disease. 2001;5(3):645-63.

4.Kim T, Shijo H, Kokawa H, Tokumitsu H, Kubara K, Ota K, et al. Risk factors for hemorrhage from gastric fundal varices. Hepatology. 1997;25(2):307-12.

5.Sarin SK, Lahoti D, Saxena SP, Murthy NS, Makwana UK. Prevalence, classification and natural history of gastric varices: a long-term follow-up study in 568 portal hypertension patients. Hepatology. 1992;16(6):1343-9.

6. Haq I, Tripathi D. Recent advances in the management of variceal bleeding. Gastroenterology report. 2017;5(2):113-26.

7.Laimer M, Pohla-Gubo G, Diem A, Prodinger C, Bauer JW, Hintner H. Epidermolysis bullosa House Austria and Epidermolysis bullosa clinical network: Example of a centre of expertise implemented in a European reference network to face the burden of a rare disease. Wiener klinische Wochenschrift. 2017;129(1-2):1-7.

8.Sarin SK, Kumar A. Endoscopic treatment of gastric varices. Clinics in liver disease. 2014;18(4):809-27.

9.Mansour L, El-Kalla F, El-Bassat H, Abd-Elsalam S, El-Bedewy M, Kobtan A, et al. Randomized controlled trial of scleroligation versus band ligation alone for eradication of gastroesophageal varices. Gastrointestinal endoscopy. 2017;86(2):307-15.

10.Sarin SK, Kumar A, Angus PW, Baijal SS, Baik SK, Bayraktar Y, et al. Diagnosis and management of acute variceal bleeding: Asian Pacific Association for Study of the Liver recommendations. Hepatology international. 2011;5(2):607-24.

11.de Franchis R, Baveno VIF. Expanding consensus in portal hypertension: Report of the Baveno VI Consensus Workshop: Stratifying risk and individualizing care for portal hypertension. Journal of hepatology. 2015;63(3):743-52.

12.Terg R, Gadano A, Cartier M, Casciato P, Lucero R, Munoz A, et al. Serum creatinine and bilirubin predict renal failure and mortality in patients with spontaneous bacterial peritonitis: a retrospective study. Liver international: official journal of the International Association for the Study of the Liver. 2009;29(3):415-9. 
13. Malinchoc M, Kamath PS, Gordon FD, Peine CJ, Rank J, ter Borg PC. A model to predict poor survival in patients undergoing transjugular intrahepatic portosystemic shunts. Hepatology. 2000;31(4):864-71.

14.Biggins SW, Kim WR, Terrault NA, Saab S, Balan V, Schiano T, et al. Evidence-based incorporation of serum sodium concentration into MELD. Gastroenterology. 2006;130(6):1652-60.

15.Reverter E, Tandon P, Augustin S, Turon F, Casu S, Bastiampillai R, et al. A MELD-based model to determine risk of mortality among patients with acute variceal bleeding. Gastroenterology. 2014;146(2):412-19 e3.

16.Seo YS. Prevention and management of gastroesophageal varices. Clinical and molecular hepatology. 2018;24(1):20-42.

17.Seo YS, Park SY, Kim MY, Kim JH, Park JY, Yim HJ, et al. Lack of difference among terlipressin, somatostatin, and octreotide in the control of acute gastroesophageal variceal hemorrhage. Hepatology. 2014;60(3):954-63.

18.Bosch J. Prevention of variceal rebleeding: endoscopes, drugs, and more. Hepatology. 2000;32(3):660-2.

19.Sung JJ, Tsoi KK, Lai LH, Wu JC, Lau JY. Endoscopic clipping versus injection and thermo-coagulation in the treatment of non-variceal upper gastrointestinal bleeding: a meta-analysis. Gut. 2007;56(10):136473.

20.Dong XJ, Chen WQ. [Therapeutic efficacy of endoscopic variceal ligation to treat gastric varices: a study of 63 consecutive cases]. Zhonghua gan zang bing za zhi = Zhonghua ganzangbing zazhi = Chinese journal of hepatology. 2013;21(9):692-6.

21.Xu L, Ji F, Xu QW, Zhang MQ. Risk factors for predicting early variceal rebleeding after endoscopic variceal ligation. World journal of gastroenterology. 2011;17(28):3347-52.

22.Kim SJ, Choi CW, Kang DH, Kim HW, Park SB, Hong YM, et al. Emergency endoscopic variceal ligation in cirrhotic patients with blood clots in the stomach but no active bleeding or stigmata increases the risk of rebleeding. Clinical and molecular hepatology. 2016;22(4):466-76.

23.Kang EJ, Jeong SW, Jang JY, Cho JY, Lee SH, Kim HG, et al. Long-term result of endoscopic Histoacryl (N-butyl-2-cyanoacrylate) injection for treatment of gastric varices. World journal of gastroenterology. 2011;17(11):1494-500.

24.Jun $\mathrm{CH}$, Kim KR, Yoon JH, Koh HR, Choi WS, Cho KM, et al. Clinical outcomes of gastric variceal obliteration using $\mathrm{N}$-butyl-2-cyanoacrylate in patients with acute gastric variceal hemorrhage. The Korean journal of internal medicine. 2014;29(4):437-44. 
25.Mishra SR, Sharma BC, Kumar A, Sarin SK. Primary prophylaxis of gastric variceal bleeding comparing cyanoacrylate injection and beta-blockers: a randomized controlled trial. Journal of hepatology. 2011;54(6):1161-7.

26.Chen WT, Lin CY, Sheen IS, Huang CW, Lin TN, Lin CJ, et al. MELD score can predict early mortality in patients with rebleeding after band ligation for variceal bleeding. World journal of gastroenterology. 2011;17(16):2120-5.

27.Elalfy H, Elsherbiny W, Abdel Rahman A, Elhammady D, Shaltout SW, Elsamanoudy AZ, et al. Diagnostic non-invasive model of large risky esophageal varices in cirrhotic hepatitis $\mathrm{C}$ virus patients. World journal of hepatology. 2016;8(24):1028-37.

28.Ying L, Lin X, Xie ZL, Hu YP, Shi KQ. Performance of platelet count/spleen diameter ratio for diagnosis of esophageal varices in cirrhosis: a meta-analysis. Digestive diseases and sciences. 2012;57(6):167281.

\section{Tables}

Table 1 The clinical features of endoscopic prophylaxis in patients with EVs combined GOV2.

\begin{tabular}{|c|c|c|c|c|}
\hline Characteristic & EVs+GOV2 $(n=124)$ & EVs+GOV2 without GOV1 $(n=76)$ & EVs+GOV2with GOV1 $(n=48)$ & P-value \\
\hline Etiology, n (\%) & & & & 0.182 \\
\hline Hepatitis Viral & $80(64.5 \%)$ & $51(67.1 \%)$ & $29(60.4 \%)$ & \\
\hline Autoimmune & $6(4.8 \%)$ & $4(5.3 \%)$ & $2(4.2 \%)$ & \\
\hline Alcoholic & $10(8.1 \%)$ & $8(10.5 \%)$ & $2(4.2 \%)$ & \\
\hline Other & $20(16.2 \%)$ & $9(11.8 \%)$ & $11(23.0 \%)$ & \\
\hline Age, median (IQR) & $49(42.25-57.50)$ & $48(41.25-59)$ & $52(43.25-55.75)$ & 0.719 \\
\hline Sex, Male (\%) & $95(76.6 \%)$ & $60(78.9 \%$ 口 & $35(72.9 \%)$ & 0.515 \\
\hline Creatinine $(\mu \mathrm{mol} / \mathrm{L})$ & $75.75(62.25-89.75)$ & $67.95(59-81.38)$ & $90(73.25-100.75)$ & 0.300 \\
\hline Bilirubin $(g / L)( \pm S D)$ & $34.10 \pm 6.48$ & $33.30 \pm 6.64$ & $35.02 \pm 6.25$ & 0.156 \\
\hline PTA (IQR) & $13.95(12.75-15.67 \square$ & $14.15(12.9-15.73 \square$ & $13.7(12.5-14.9)$ & 0.125 \\
\hline INR (IQR) & $1.21(1.11-1.35)$ & $1.21(1.11-1.39)$ & $1.21(1.10-1.30)$ & 0.355 \\
\hline Albumin $(\mu \mathrm{mol} / \mathrm{L})(\mathrm{IQR})$ & $16.85(11.53-24.69)$ & $16.65(11.72-25.93)$ & $18.15 \square 11.32-22.95)$ & 0.949 \\
\hline Hemoglobin $(\mathrm{g} / \mathrm{L})( \pm \mathrm{SD})$ & $87.89 \pm 23.53$ & $88.41 \pm 25.63$ & $86.40 \pm 21.30$ & 0.651 \\
\hline Platelet count $\left(\times 10^{9} / \mathrm{L}\right)(\mathrm{IQR})$ & $69.6(43.25-99.25)$ & $66.5(42-96.75)$ & $73.5(44.75-102)$ & 0.416 \\
\hline MELD (IQR) & $9.15(7.54-10.87)$ & $9.28(7.62-11.18)$ & $8.82(7.47-10.7)$ & 0.376 \\
\hline MELD-Na (IQR) & $9.34(7.72-11.75)$ & $9.97(7.96-12.50)$ & $8.86(7.53-10.90)$ & 0.238 \\
\hline Child-Pugh (A/B/C) & $49 / 63 / 12$ & $26 / 42 / 8$ & $23 / 21 / 4$ & 0.315 \\
\hline $\mathrm{HE}, \mathrm{n}(\%)$ & $5(4.0 \%)$ & $3(3.9 \%)$ & $2(4.2 \%)$ & 0.998 \\
\hline HCC, n (\%) & $13(10.4 \%)$ & $9(11.8 \%)$ & $4(8.4 \%)$ & 0.765 \\
\hline Ascites (no/mild/severe) & $56 / 31 / 37$ & $32 / 21 / 23$ & $24 / 10 / 14$ & 0.618 \\
\hline Renal impairments, n (\%) & $2(1.6 \%)$ & $1(1.3 \%)$ & $1(2.1 \%)$ & 0.994 \\
\hline Splenectomy, n (\%) & $16(12.9 \%)$ & $10(13.2 \%)$ & $6(12.6 \%)$ & 0.893 \\
\hline SBP, n (\%) & $3(2.4 \%)$ & $1(1.3 \%)$ & $2(4.2 \%)$ & 0.320 \\
\hline
\end{tabular}

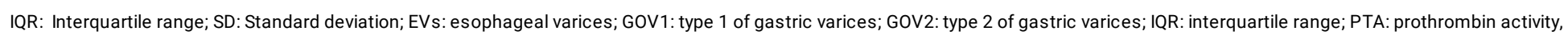
INR: international normalized ratio; MELD: model for end-stage liver disease; MELD-Na: MELD combined with serum sodium concentration; HE: hepatic encephalopathy; HCC: 
Table 2 The endoscopic features and treatment details of endoscopic prophylaxis in patients with EVs combined GOV2

\begin{tabular}{|c|c|c|c|c|}
\hline Characteristics & EVs+GOV2 $(n=124)$ & EVs+GOV2 without GOV1 $(n=76)$ & EVs+GOV2 with GOV1 $(n=48)$ & P-value \\
\hline Red spot, n (\%) & $111(89.5 \%)$ & $65(85.5 \%)$ & $46(95.8 \%)$ & 0.079 \\
\hline Communicating branch, n (\%) & $107(86.3 \%)$ & $63(82.9 \%)$ & $44(91.7 \%)$ & 0.192 \\
\hline Active bleeding at endoscopy, n (\%) & $32(25.8 \%)$ & $19(25 \%)$ & $13(27.1 \%)$ & 0.796 \\
\hline Blood clots, n (\%) & $25(20.2 \%)$ & $17(22.4 \%)$ & $8(16.7 \%)$ & 0.441 \\
\hline variceal diameter $(\mathrm{cm})(\mathrm{IQR})$ & $1.23(1.05-1.62)$ & $1.2(1.2-1.5)$ & $1.25(0.85-1.75)$ & 0.103 \\
\hline Varices distance from the incisors $(\mathrm{cm})$ & $20.16 \pm 5.93$ & $20.01 \pm 6.00$ & $20.27 \pm 5.86$ & 0.815 \\
\hline $\mathrm{EBL}, \mathrm{n}(\%)$ & $95(76.6 \%)$ & $62(81.6 \%)$ & $33(68.8 \%)$ & \\
\hline Use 2 pairs of legators, n (\%) & $30(24.2 \%)$ & $22(35.5 \%)$ & $8(24.2 \%)$ & 0.203 \\
\hline Number of rubber bands, $( \pm S D)$ & $8.1 \pm 2.9$ & $8.6 \pm 3$ & $7.6 \pm 2.7$ & 0.109 \\
\hline EVS+EVO, n (\%) & $11(8.9 \%)$ & $5(6.6 \%)$ & $6(12.5 \%)$ & \\
\hline Injection sessions (IQR) & $4(2.6-6)$ & $4(3-5)$ & $4(1.5-7)$ & 0.968 \\
\hline Sclerotherapy dose (mL) (IQR) & $34(25-60)$ & $34(25-60)$ & $30(17.5-54)$ & 0.537 \\
\hline Cyanoacrylate dose (mL) (IQR) & $3(2-2.5)$ & $3(2-2.5)$ & $2.75(1.37-4)$ & 0.989 \\
\hline EBL+EVS+EVO, n (\%) & $6(4.8 \%)$ & $2(2.6 \%)$ & $4(8.3 \%)$ & \\
\hline EBL+EVO, n (\%) & $3(2.4 \%)$ & $1(1.3 \%)$ & $2(4.2 \%)$ & \\
\hline EBL+EVS, n (\%) & $1(0.8 \%)$ & 0 & $1(2.1 \%)$ & \\
\hline EVO, n (\%) & $2(2.6 \%)$ & $1(1.3 \%)$ & $1(2.1 \%)$ & \\
\hline EVS, n $(\%)$ & $6(4.8 \%)$ & $5(6.7 \%)$ & $1(2.1 \%)$ & \\
\hline
\end{tabular}

IQR: interquartile range; EVs: endoscopic variceal sclerotherapy; EBL: endoscopic band ligation; EVO: endoscopic variceal obturation.

Table 3 Comparing clinical features, endoscopic features and treatment details of endoscopic prophylaxis between rebleeding and non-bleeding groups. 


\begin{tabular}{|c|c|c|c|}
\hline Characteristics at enrollment & Rebleeding within 1-year $(\mathrm{n}=11)$ & Non-rebleeding within 1-year $(\mathrm{n}=92)$ & P-value \\
\hline EVs+GOV2 with GOV1, n (\%) & $7(55.6 \%)$ & $30(37.5 \%)$ & 0.043 \\
\hline Red spot, n (\%) & $72(78.2 \%)$ & $8(72.7 \%)$ & 0.677 \\
\hline communicating branch, n (\%) & $79(85.8 \%)$ & $8(72.7 \%)$ & 0.255 \\
\hline Active bleeding at endoscopy, n (\%) & $6(54.5 \%)$ & $28(30.4 \%)$ & 0.108 \\
\hline Blood clots, n (\%) & $3(27.3 \%)$ & $20(21.7 \%)$ & 0.677 \\
\hline Variceal diam eter $(\mathrm{cm})(\mathrm{IQR})$ & $1.2(0.8-1.5)$ & $1.2(0.85-1.75)$ & 0.814 \\
\hline Varices distance from the incisors $(\mathrm{cm})( \pm \mathrm{SD})$ & $22.11 \pm 5.56$ & $19.78 \pm 5.88$ & 0.255 \\
\hline Endoscopic treatment, n (\%) & & & 0.182 \\
\hline EBL & $72(78.2 \%)$ & $9(81.8 \%)$ & \\
\hline $\mathrm{EVS}+\mathrm{EVO}$ & $6(6.5 \%)$ & $2(18.2 \%)$ & \\
\hline Others & $14(15.2 \%)$ & 0 & \\
\hline Etiology (\%) & & & 0.796 \\
\hline Hepatitis Viral, n (\%) & $67(72.8 \%)$ & $8(72.7 \%)$ & \\
\hline Autoimmune, n (\%) & $6(6.5 \%)$ & 0 & \\
\hline Alcoholic, n (\%) & $8(8.7 \%)$ & $1(9.1 \%)$ & \\
\hline Other, n (\%) & $11(11.9 \%)$ & $2(18.2 \%)$ & \\
\hline Age, median (IQR) & $64(47-72)$ & $48.5(42-56)$ & 0.039 \\
\hline Sex, Male (\%) & $72(78.3 \%)$ & $7(63.6 \%)$ & 0.278 \\
\hline $\mathrm{Na}(\mathrm{mmol} / \mathrm{L})(\mathrm{IQR})$ & $139.1(137-141)$ & $139(136.95-140.95)$ & 0.718 \\
\hline Creatinine $(\mu \mathrm{mol} / \mathrm{L})(\mathrm{IQR})$ & $67.35(56.55-78.15)$ & $57.5(50.4-73.3)$ & 0.311 \\
\hline Albumin $(g / L)$ & $31.07 \pm 7.75$ & $34.49 \pm 6.31$ & 0.128 \\
\hline PTA (IQR) & $15.6(13.7-20.45)$ & $13.9(12.52-14.97)$ & 0.019 \\
\hline INR (IQR) & $1.3(1.17-1.56)$ & $1.21(1.11-1.33)$ & 0.132 \\
\hline Bilirubin $(\mu \mathrm{mol} / \mathrm{L})(\mathrm{IQR})$ & $36.2(13.75-64.4)$ & $16.4(11.23-22.35)$ & 0.033 \\
\hline Hem oglobin (g/l) (IQR) & $88.41 \pm 25.63$ & $80.11 \pm 25.26$ & 0.297 \\
\hline Platelet count $\left(\times 10^{9} / \mathrm{L}\right)(\mathrm{IQR})$ & $65(42.25-96)$ & $80(40-106.5)$ & 0.041 \\
\hline Child-Pugh A/B/C & $30 / 52 / 10$ & $2 / 4 / 5$ & 0.009 \\
\hline MELD (IQR) & $13.47(7.77-15.93)$ & $8.85(7.68-10.64)$ & 0.044 \\
\hline MELD-Na (IQR) & $13.47(7.77-17.22)$ & $9.16(7.96-11.23)$ & 0.090 \\
\hline $\mathrm{HE}, \mathrm{n}(\%)$ & $2(18.2 \%)$ & $3(3.3 \%)$ & 0.197 \\
\hline HCC, n (\%) & $11(12.0 \%)$ & $1(9.1 \%)$ & 0.799 \\
\hline Ascites (no/mild/severe $\square$ & $37 / 31 / 24$ & $4 / 2 / 5$ & 0.353 \\
\hline Renal impairment, n (\%) & $2(2.2 \%)$ & 0 & 0.621 \\
\hline Splenectomy, n (\%) & $2(18.2 \%)$ & $13(14.1 \%)$ & 0.719 \\
\hline SBP, n (\%) & $1(9.1 \%)$ & $2(2.2 \%)$ & 0.197 \\
\hline
\end{tabular}

IQR: Interquartile range; SD: Standard deviation;EVs: esophageal varices; GOV1: type 1 of gastric varices; GOV2: type 2 of gastric varices; IQR: interquartile range; EVs: endoscopic variceal sclerotherapy; EBL: endoscopic band ligation; EVO: endoscopic variceal obturation; PTA: prothrombin activity, INR: international normalized ratio; MELD: model for end-stage liver disease; MELD-Na: MELD combined with serum sodium concentration; HE: hepatic encephalopathy; HCC: Hepatocellular carcinoma; SBP: spontaneous peritonitis

Table 4 Multivariate analyses of risk factors associated with rebleeding for 1-year

\begin{tabular}{lccccc}
\hline Characteristics & $\beta$ & $\mathrm{SE}$ & $\mathrm{P}$ & $\mathrm{OR}$ & $95 \% \mathrm{CI}$ \\
\hline Age, (years) & 0.170 & 0.062 & 0.006 & 1.186 & $1.050-1.340$ \\
Variceal classification & 3.436 & 1.526 & 0.024 & 3.063 & $1.560-6.184$ \\
& & & & & \\
(EVs+GOV2 with GOV1) & & & & & \\
Bilirubin, ( $\mathrm{mmol} / \mathrm{L})$ & 0.084 & 0.032 & 0.009 & 1.088 & $1.022-1.158$ \\
PTA & 0.103 & 0.077 & 0.038 & 1.109 & $1.042-1.288$ \\
\hline
\end{tabular}




\section{Figures}

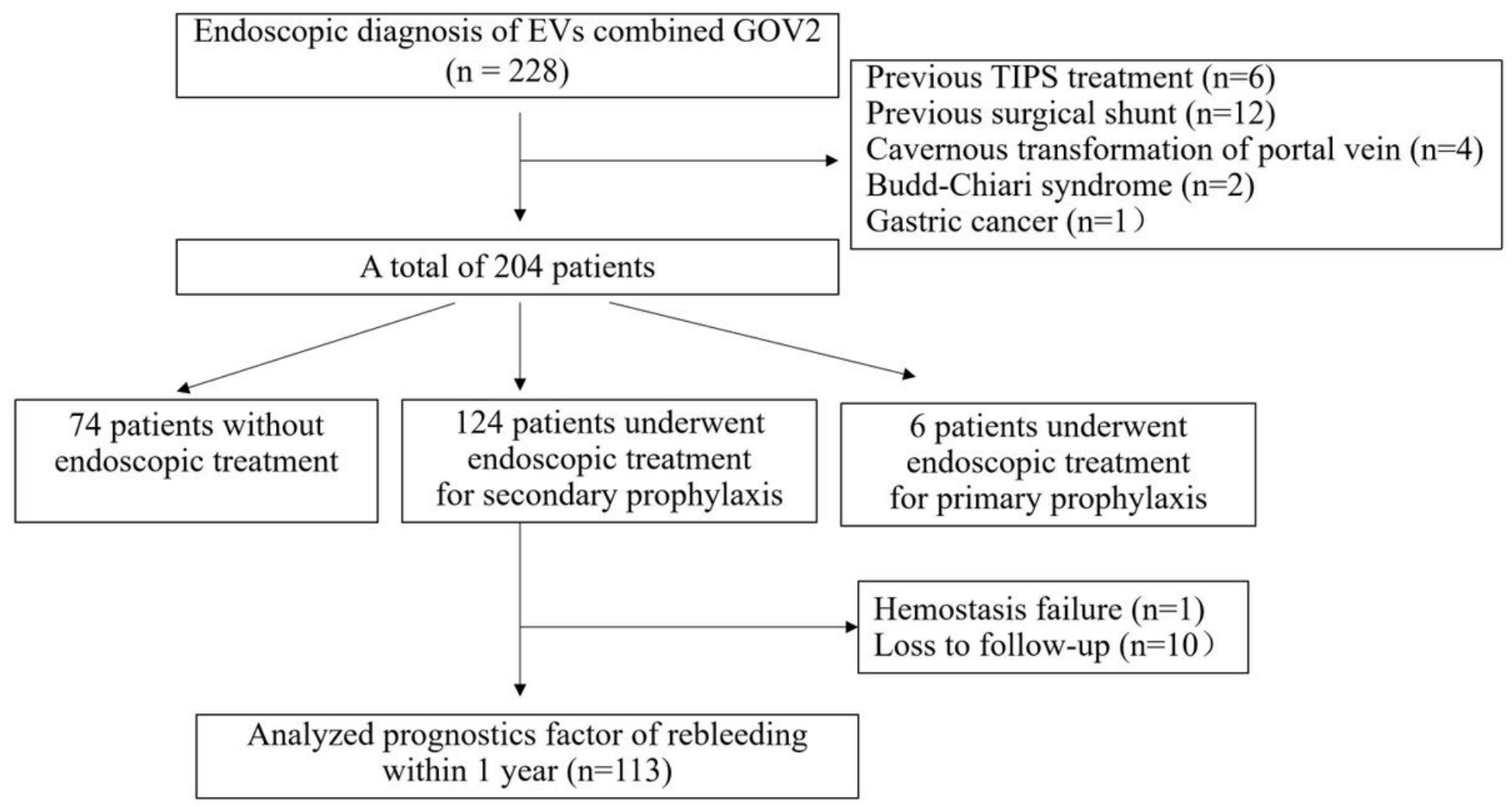

\section{Figure 1}

Study flow diagram showing each stage of inclusion, exclusion, and loss to follow-up of patients in our study. 